\title{
Inflatable Re-entry Vehicle Experiment (IRVE) - 4 Overview
}

\author{
Daniel K. Litton ${ }^{1}$ \\ NASA Langley, Hampton, VA, 23681 \\ David M. Bose ${ }^{2}$ \\ Analytical Mechanics Associates, Plano, TX, 75024 \\ F. McNeil Cheatwood ${ }^{3}$, Stephen Hughes ${ }^{4}$, Henry S. Wright ${ }^{5}$, Michael C. Lindell ${ }^{6}$, and Stephen D. Derry ${ }^{7}$ \\ NASA Langley, Hampton, VA, 23681 \\ Aaron Olds ${ }^{8}$ \\ Analytical Mechanics Associates, Hampton, VA, 23681
}

\begin{abstract}
The suite of Inflatable Re-Entry Vehicle Experiments (IRVE) is designed to further our knowledge and understanding of Hypersonic Inflatable Aerodynamic Decelerators (HIADs). Before infusion into a future mission, three challenges need to be addressed: surviving the heat pulse during re-entry, demonstrating system performance at relevant scales, and demonstrating controllability in the atmosphere. IRVE-4 will contribute to a better understanding of controllability by characterizing how a HIAD responds to a set of controlled inputs. The ability to control a HIAD is vital for missions that are g-limited, require precision targeting and guidance for aerocapture or entry, descent, and landing. The IRVE-4 flight test will focus on taking a first look into controlling a HIAD. This paper will give an overview of the IRVE-4 mission including the control response portion of the flight test sequence, and will provide a review of the mission's development.
\end{abstract}

\section{Introduction}

$\mathrm{C}$ urrent planetary entry vehicles are reaching the size limits on launch vehicle fairings. Entry vehicles are also reaching the limit on the amount of mass that can be decelerated and safely reach the planets surface. The science payload size and site altitude are limited by Viking EDL architecture. Mars Science Laboratory has taken the Viking heritage system about as far as possible in terms of landed mass and altitude. ${ }^{1}$ At Mars, in particular the thin atmosphere makes it difficult to decelerate large masses in addition to limiting the surface altitudes that can be reached. The inflatable aeroshell/heatshield enables the payload and entry vehicle to grow greatly. Hypersonic Inflatable Aerodynamic Decelerators (HIADS) enable a reduction in the ballistic coefficient of an entry vehicle through an increase in drag area in exchange for a minimal mass impact compared to a rigid vehicle of the same size. This reduction in ballistic coefficient enables deceleration at higher altitudes allowing access to higher elevations, reduced heat rates, an increase in delivered payload, and timeline margin.

There are three overarching challenges that the HIAD program has to address to ensure proper infusion into future missions. These three challenges are surviving the heat pulse during atmospheric entry, demonstrating system performance at relevant scales, and demonstrating controllability in the atmosphere. The HIAD program will address these three challenges through 4 missions, namely IRVE-3, IRVE-4, and the High-Energy Atmospheric Reentry Test (HEART and HEART-2). IRVE II was a technology demonstration mission that confirmed the

\footnotetext{
${ }^{1}$ Study Lead, Atmospheric Flight and Entry Systems Branch, Senior Member.

${ }^{2}$ Vice President, Modeling and Simulation, , AIAA Senior Member.

${ }^{3}$ Principal Investigator, Atmospheric Flight and Entry Systems Branch, AIAA Associate Fellow.

${ }^{4}$ HIAD Chief Engineer, Mechanical Systems Branch, AIAA Member.

${ }^{5}$ IRVE-4 Principal Investigator, Atmospheric Flight and Entry Systems Branch, AIAA Senior Member.

${ }^{6}$ Lead Structural Analyst, Structural \& Thermal Systems Branch, NASA LaRC, AIAA Member.

${ }^{7}$ Research Aerospace Engineer, Dynamic Systems and Control Branch.

${ }^{8}$ Flight Dynamics Lead, Atmospheric Flight and Entry Systems Branch.
}

1

American Institute of Aeronautics and Astronautics 
feasibility of using HIADs from the standpoint of inflating the aeroshell exo-atmospherically and maintaining pressure during the pressure pulse in addition to demonstrating re-entry survivability at a heating of $2 \mathrm{~W} / \mathrm{cm}^{2}$. IRVE-3 will increase the heating to more than $15 \mathrm{~W} / \mathrm{cm}^{2}$ and demonstrate the effect of lift on a HIAD's trajectory performance. IRVE-3 will not perform any maneuvers during the experiment window (re-entry through Mach 0.7), but rather will maintain a zero bank angle.

HEART is currently planned to be have orbital velocities demonstrating the whole system for a relevant-scale HIAD in relevant environments. HEART will be ballistic (unguided) and will show that the HIAD can perform on the scale of 8-9 meters. HEART-2 will be of a similar nature, except it will have the ability to control the HIAD to land at a targeted location. The HIAD's lift vector will be used to maneuver through the atmosphere to its targeted location. In order to successfully complete this mission and bridge the gap between HEART and HEART-2, IRVE4 will take a buy down the risk on a costly mission by taking a first look into the HIADs response to a set of control inputs on an inexpensive sub-scale vehicle.

The flexibility of a HIAD makes this vehicle unique when compared to its rigid counterparts such as the Viking and ORION/Apollo capsules. This flexibility comes about from two places. The first is that the aeroshell may be able to flex and change shape in flight as the dynamic pressure increases. This could potentially alter the shape of the toroids from circular to elliptical. The other unique feature is that the aeroshell connection to the centerbody, where the payload is stored, is not rigid. Larger dynamic pressures could potentially cause the aeroshell to weathervane and be at a different angle than the centerbody. All of these unique features call for a better understanding of how these vehicles react in flight to a set of control inputs. IRVE-4 will bring to the forefront some of the major risks that come with controling a HIAD before having to deal with these on a large-scale vehicle.

Having the ability to control a HIAD through redirecting lift opens a vast array of missions. Lift opens up a whole set of tools and abilities for entry vehicles such as the reduction of the entry vehicle's deceleration environment, mitigation of environmental uncertainties such as wind and density, improved landing accuracy, and allows larger approach navigation uncertainty. Additionally, having the ability to control a HIAD allows the opportunity to perform a non-propulsive orbital plane change and access to higher surface elevations by increasing the altitude at staging for the next decelerator. Most, if not all of these characteristics are important to potential future crewed and uncrewed vehicles.

The current plan is to use IRVE-4 to understand two different types of control. The first method of control will use bank control, where the lift vector is banked/rotated around to steer through the atmosphere by changing the downrange and crossrange. The other method of altering lift is to directly change the amount of lift the vehicle has by moving the lateral center of gravity (CG) back and forth. The further from the centerline the offset CG is the more lift the vehicle will produce. This moving offset CG in combination with a Reaction Control System (RCS) will not only enable the lift vector direction to be altered, but also the magnitude of that lift vector to be changed in flight. In addition to investigating these two types of control, parameter identification maneuvers (PID) will be placed on top of and/or around the control maneuvers in order to back out aerodynamics and potentially structural modes of a HIAD. These predetermined set of maneuvers will be designed to back out the aerodynamics and control responses that cannot be determined through ground testing. These maneuvers will be performed by either bank control with a fixed CG offset or through a combination of moving the CG and firing the ACS thrusters. Of particular interest from this experiment will be the vehicles dynamic responses such as overshoots and response lags when commanded to go from full lift up to full lift down.

\section{Background}

As mentioned in the Introduction, the goal of the IRVE-4 flight test is to characterize HIAD response to control inputs. This will be achieved through demonstration of two types of control methodologies and through the identifcation of parameters key to the accurate modeling of vehicle response. This approach will provide an immediate confirmation of the mechanisms required to perform closed loop guidance of entry vehicles of this class, in particular the ability to impart a change in trajectory downrange and crossrange through redirecting the lift vector and/or the modulation of lift-to-drag ratio through bank angle or CG control. It will also provide the data necessry to validate vehicle response models; allowing more detailed evaluation of control methodlogies and their extension to candidate missions and their associated environments.

To this end, several characteristics are important to guidance and controls engineers including control authority (CL/CD), pitch stability $\left(\mathrm{C}_{\mathrm{m}} \alpha\right)$, and dynamic stability $\left(\mathrm{C}_{\mathrm{mq}}\right)$. Estimation of these parameters must be performed in consideration of a number of factors including atmospheric uncertainty, mass properties (particularly in the case of CG modulation), and NIACS contributions to measured forces and moments. 
An additional factor important to the accurate modeling of vehicle response is flexibility. Axdahl et. al [2009] ${ }^{2}$ demonstrated the potential impacts of flexibility through numerical simulation of a two-body entry vehicle comprised of an IAD attached to a rigid aeroshell with a flexible connection. Of particular note in Axdahl's results was the identification of three zones of dynamic stability, which depended on the level of stiffness and damping assumed in the connection. For a bare-airframe $\mathrm{C}_{\mathrm{mq}}>0$, these three zones and the qualitative stiffness and damping of the connection include 1) divergent (low stiffness), 2) statically and dynamically stable (moderate stiffness and moderate damping), and statically stable but dynamically unstable (high stiffness).

Aspects of the dynamics presented in Axhdahl [2009] ${ }^{2}$ were experienced in the IRVE-II flight test. O'Keefe et al $[2010]^{3}$ presented the post-flight trajectory reconstruction results of IRVE-II. This included a comparison of flight performance with pre-flight predictions. These comparisons brought to light a few discrepancies between simulation-based predictions and flight perforance. Two examples are illustrated in Figure 2.1, which includes a) an unexpected transient in angle of attack, and b) normal acceleration far outside pre-flight bounds.
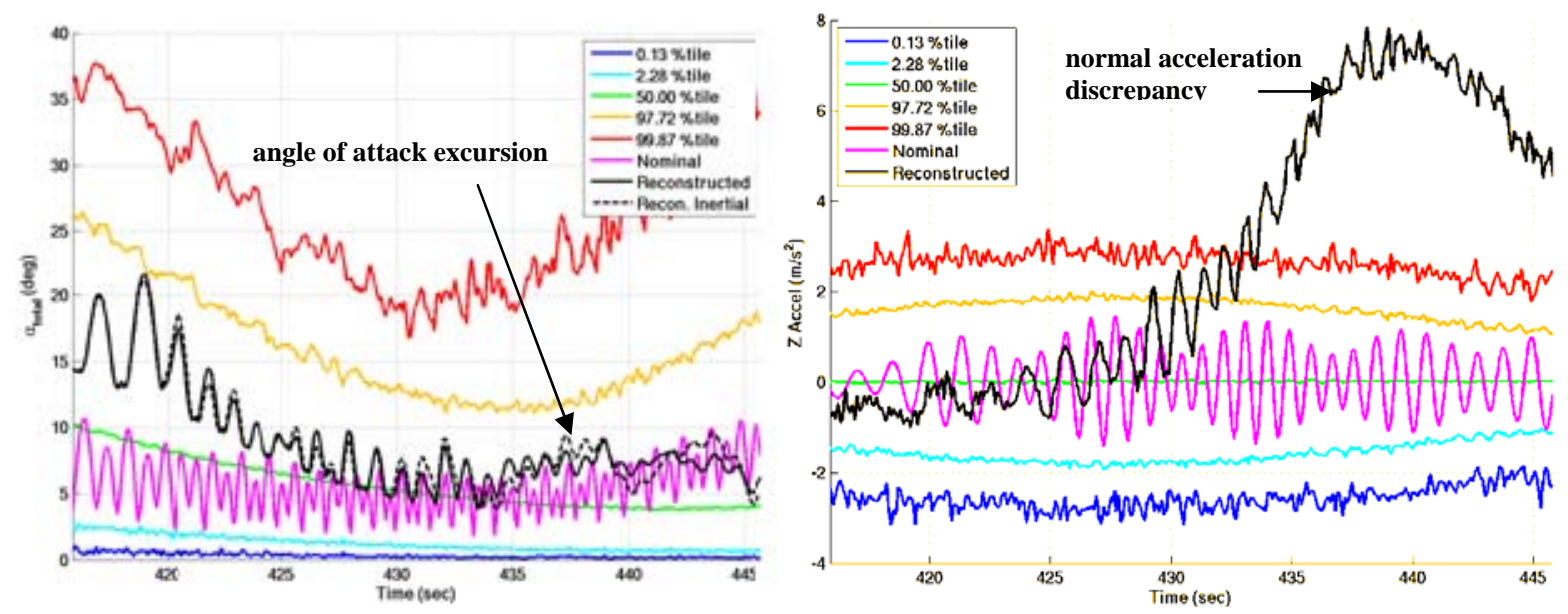

Figure 2.1: Flight Performance vs. Pre-flight Nominal and Monte Carlo Predictions a) Total Angle of Attack, b) Normal Acceleration

O'Keefe also points out that flexibility of the IRVE-II aeroshell may account for these characteristics. Flight video indicates that the aeroshell deflected, relative to the centerbody, during the pressure pulse. Subsequent analysis of the IRVE-II flight data has estimated the magnitude of deflection (approximately 3 to 5 degrees) and confirmed its impact on flight dynamics. Interestingly, as the aerohsell deflects the composite vehicle (aeroshell + centerbody) develops a lateral CG offset. In turn, this CG offset causes an increase in the trim angle of attack. As a result, the vehicle flies at a higher angle of attack than one would expect from a rigid equivalent. As Axdahl points out, if the stiffness is low enough the vehicle will actually depart.

Based on the IRVE-II experience, and considering how this may translate to full scale vehicles, the potential impacts of flexibility need to be cpatured; both in terms of the impact to post-flight data reduction and to the accurate modeling of vehicle reponse. This provides additional motivation for the IRVE-4 flight test. As seen in Section VI of this paper, flexibility will be accounted for both in terms of PID maneuver design and planned postflight data analysis methods.

\section{Vehicle Overview}

IRVE-4 as currently planned will have a 3-meter diameter, 60 deg sphere-cone aeroshell and will be a near rebuild of IRVE-3. IRVE-4 will fly on a Black Brant X (BBX) out of Wallops Flight Facility (WFF). Due to its ability to shallow out the reentry flight path angle by firing the $3^{\text {rd }}$ stage exo-atmospherically, the BBX enables an extended duration pressure pulse where flight conditions will not change as rapidly. This extended flight duration after reentry affords the time to perform the required set of maneuvers to investigate the HIADs response to control inputs. The results of IRVE-4 flight test will aid greatly in validating structural and aerodynamic models for use on future missions.

\section{Launch Vehicle}


One challenge with IRVE-4 is that the Mach number will be changing rapidly during the experiment window. A longer experiment window helps in two ways: a longer experiment window enables the ability to perform more, or longer maneuvers, a larger experiment window also makes the change in Mach number less rapid. In order to accomplish the longer time-line, IRVE-4 has to depart from the Launch Vehicle that IRVE-3 is planning to use. IRVE-3 plans to use the Black Brant XI. Unfortunately, the combination of not being able to fire the $3^{\text {rd }}$ stage above $50,000 \mathrm{ft}$ and the launch elevation no less than 79 degrees only gives an experiment window that ranges from 2735s. The Black Brant X, although not as powerful as the Black Brant XI, allows the launch rail to go down to 75 degrees and the third stage to be fired exo-atmospherically matches well with what IRVE-4's requirements. The experimental window now ranges from 53-65s, or nearly double what the Black Brant XI offers. The payload on the Black Brant $X$ is composed of the reentry vehicle itself, the transition segment from the $3^{\text {rd }}$ stage to the reentry vehicle, and the nose cone. Figure 3.1 shows the breakout of these components.

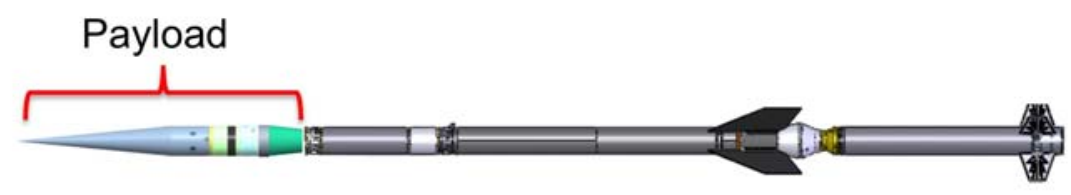

Figure 3.1: Black Brant X with IRVE-4 Payload

\section{Concept of Operations}

The sequence of events leading up the the IRVE-4 experiment are shown in Figure 3.2. As previously mentioned, the Black Brant $\mathrm{X}$ is responsible for getting IRVE-4 to the required test conditions.

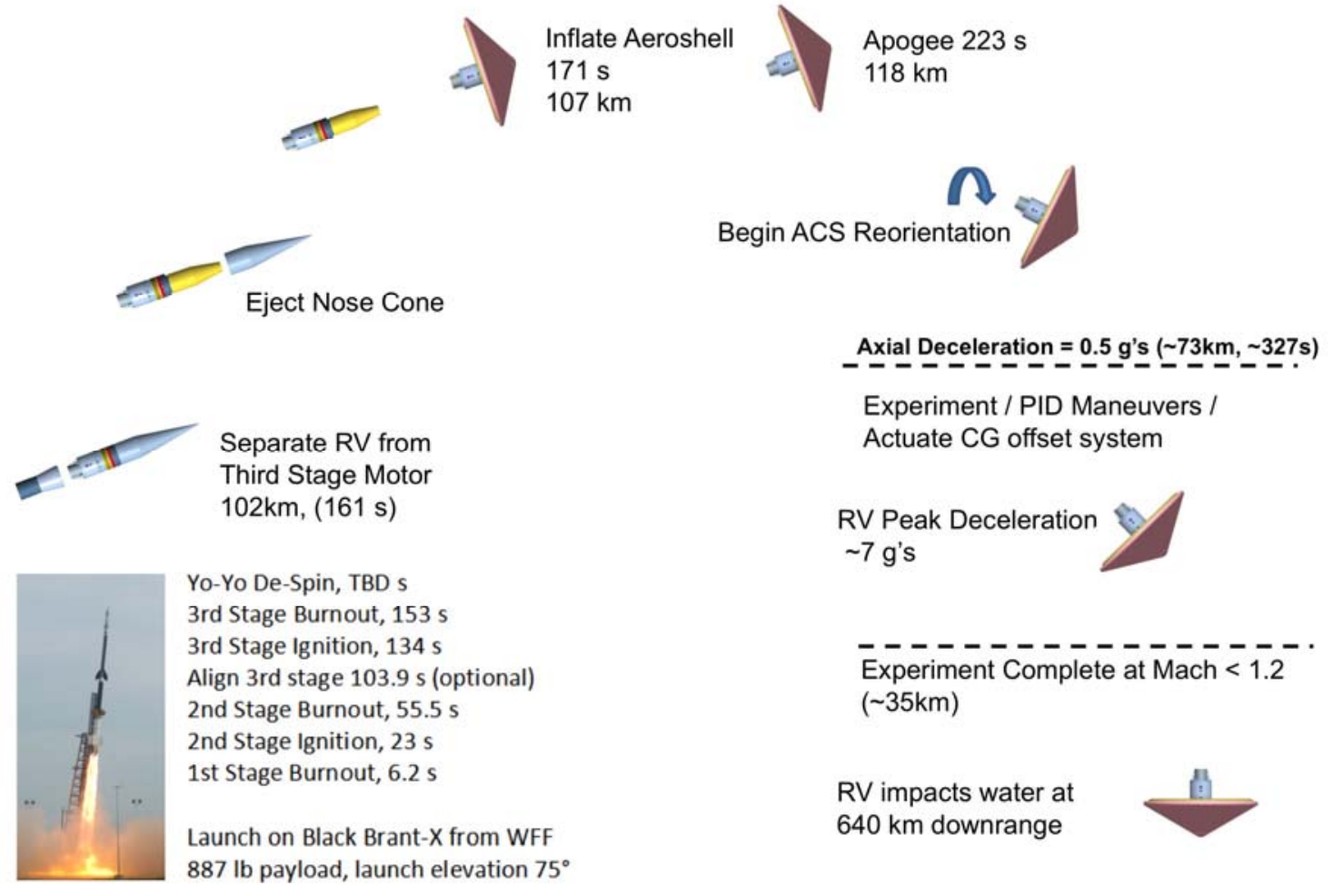

Figure 3.2: Concept of Operations

After the Black Brant X's third stage releases the Re-entry Vehicle and the nose cone has been ejected, the aeroshell inflates exo-atmospherically. Apogee is reached while the aeroshell is still being inflated. Either after or during inflation the NSROC Inertial Attitude Control System (NIACS) will orient the vehicle to a suitable attitude for reentry. The experiment will last approximately 60 seconds, starting when the vehicle begins decelaration at $0.5 g$ 's 
and slows down to Mach 1.2. An important thing to note is that the experiment starts around $73 \mathrm{~km}$ and ends around $35 \mathrm{~km}$. Using the radar and telemetry systems both at Wallop's Flight Facility and their alternate location at Coquina, loss of signal will occur around 47km. Because IRVE-4's experiment ends around 35km a sea or air asset will be utilized to telemeter the data to the ground.

\section{Re-entry Vehicle}

The reentry vehicle is composed of 6 different segments as shown in figure 3.4. The aeroshell and Thermal Protection System, Telemetry Section, inflation system segment, CG offset mechanism, ballast section, and NIACS segment. The total mass of all components of the RV currently stands at $630 \mathrm{lbs}$.
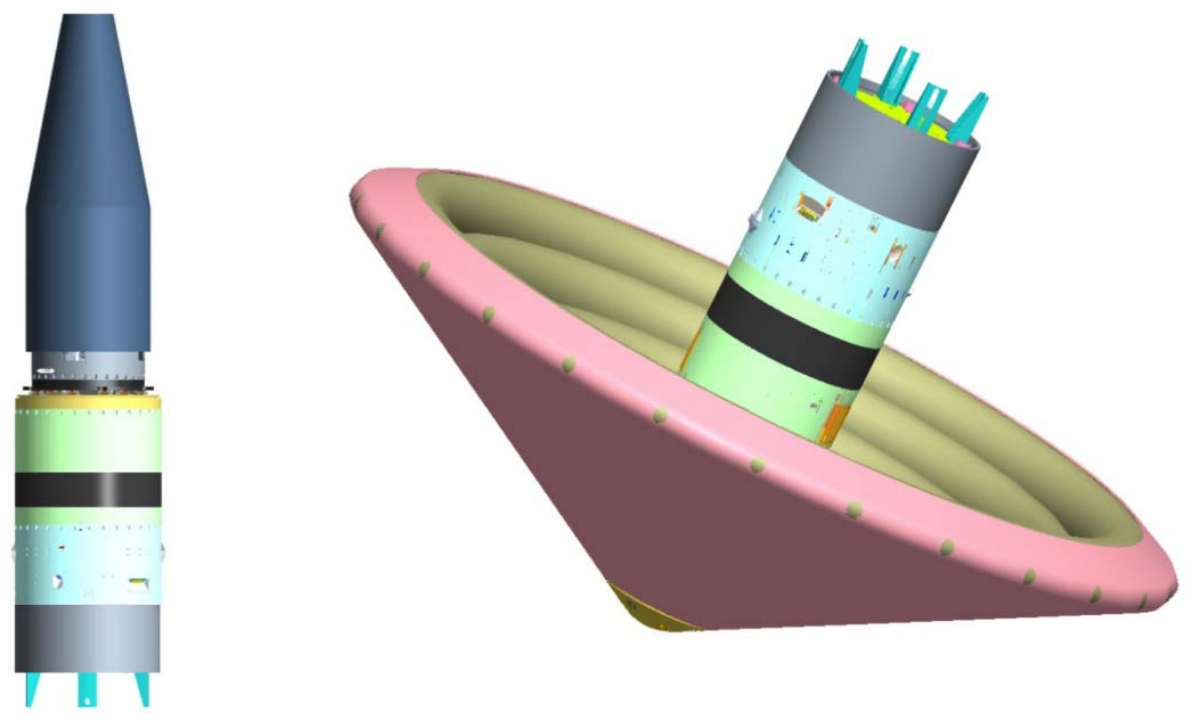

Figure 3.3: Stowed and Deployed IRVE-4 vehicle

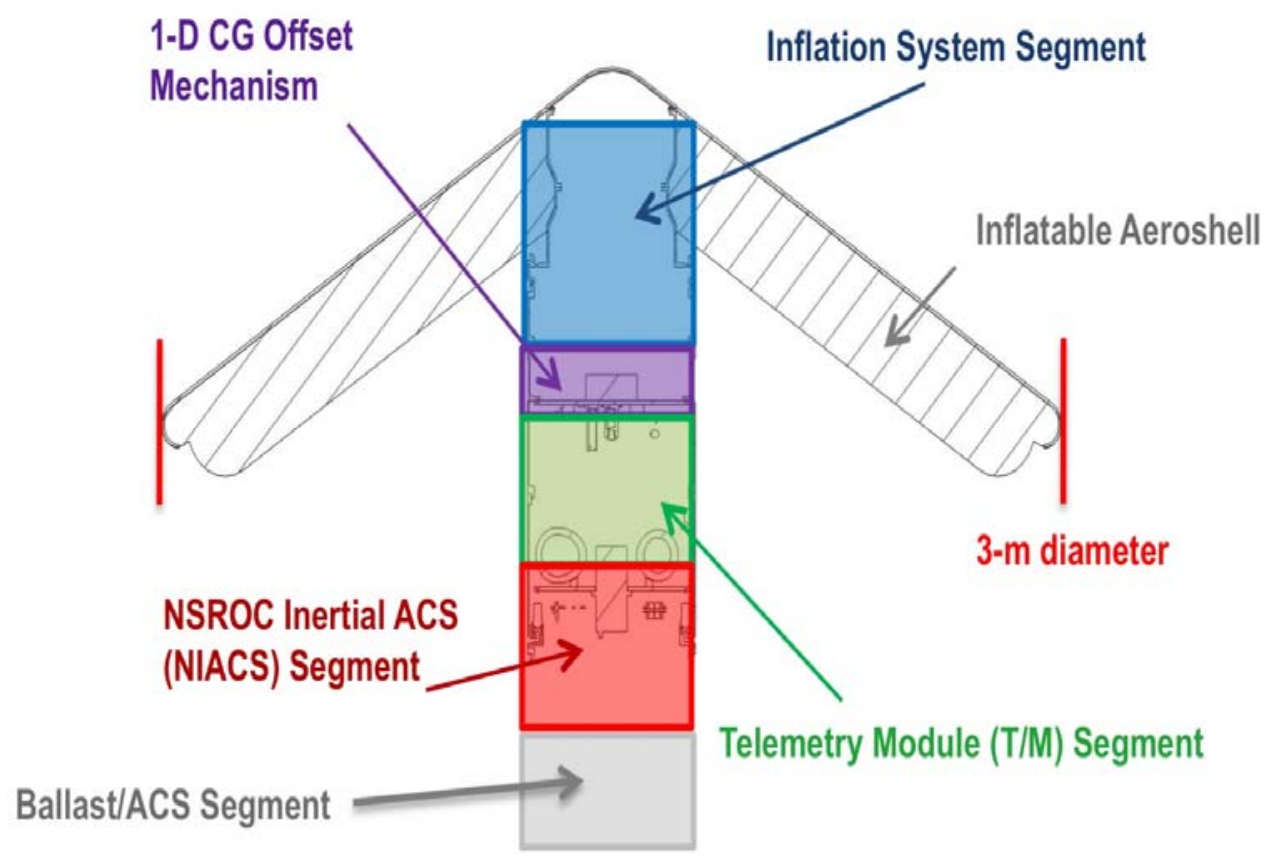

Figure 3.4: Layout of IRVE-4 Reentry Vehicle

American Institute of Aeronautics and Astronautics 
The aeroshell, shown in figure 3.5 is composed of 7 toroids filled with Nitrogen. Kevlar provides the structural strength and an overwrap of silicone prevents the Nitrogen from leaking outside the toroid. The aeroshell is initially inflated exo-atmospherically by the inflation system segment and then maintains a set pressure as the RV descends through the atmosphere. The red straps in Figure 3.5 are structural to maintain shape during flight. The blue straps are also structural but also connect the toroids to the centerbody. The TPS, shown in Figure 3.6 consists of 3 layers that protect the toroids from reentry heating. The outer fabrics deflect or absorb the initial heat load and the shearing force from the flow. The insulating layers reduce the temperature to keep the gas barrier and toroids below their respective maximum operating temperatures. Finally, the laminated structural/gas barrier provides an interface point (Kevlar) to the inflatable structure, in addition to providing a place to quilt the TPS assembly together. The Kapton portion of this final barrier is impermeable and serves to deadhead the flow reducing, or preventing the hot gasses from passing through to the toroids. The three TPS layers stretch across the whole frontal area, or windward side of the vehicle and attach just behind the smaller $7^{\text {th }}$ toroid.
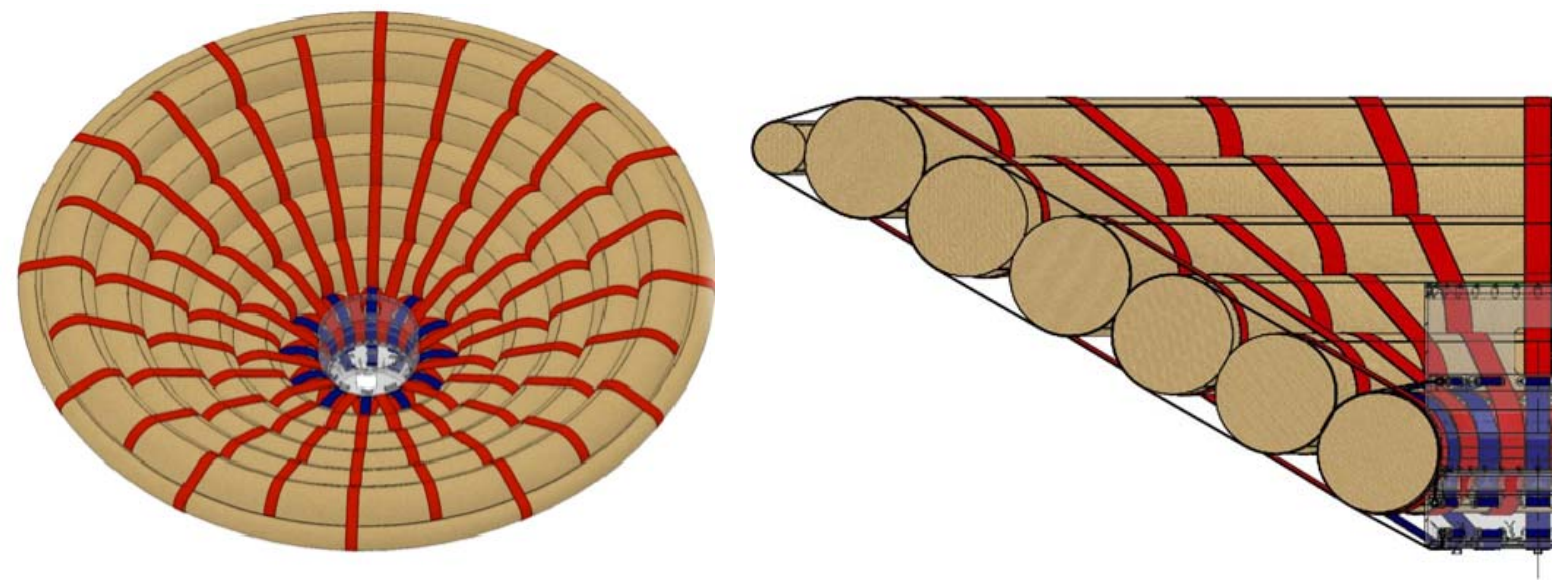

Figure 3.5: IRVE-4 Aeroshell Toroid Layout and Strap Configuration

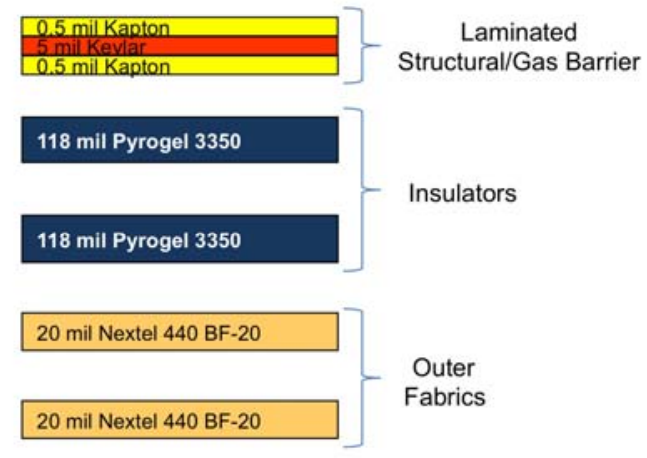

Figure 3.6: Thermal Protection Layout for IRVE-4 Re-entry Vehicle

The inflation segment is responsible for initially inflating the aeroshell exo-atmospherically and maintaining pressure during atmospheric entry. The inflation segment consists of a Nitrogen tank initially kept at 3000 psi, one control valve, and two manifolds connecting all eight (one toriod is filled by both manifolds) fill lines to the toroids. Additionally, three pairs of two toroids are connected/ganged together to aid the seven toroids in reaching system equilibrium faster. During fill, when any one toroid reaches the set pressure, the control valve shuts off for all toroids. Because the toroids all fill at different rates, the higher pressure toriods will equilibrate with the lower toroid(s) due to being ganged together and through the manifolds. An example pressure profile for the nominal IRVE-4 mission is given in Figure SSS. The spikes are due to the first toroid reaching the set point, or 14 psig. The rise in atmospheric pressure, rather than the toroids leaking, is the main contributor to the decrease in gauge pressure. As can be seen with Figure 3.7, the experiment ends at 217s, leaving extra gas in the system to potentially perform maneuvers after the experiment has concluded. 

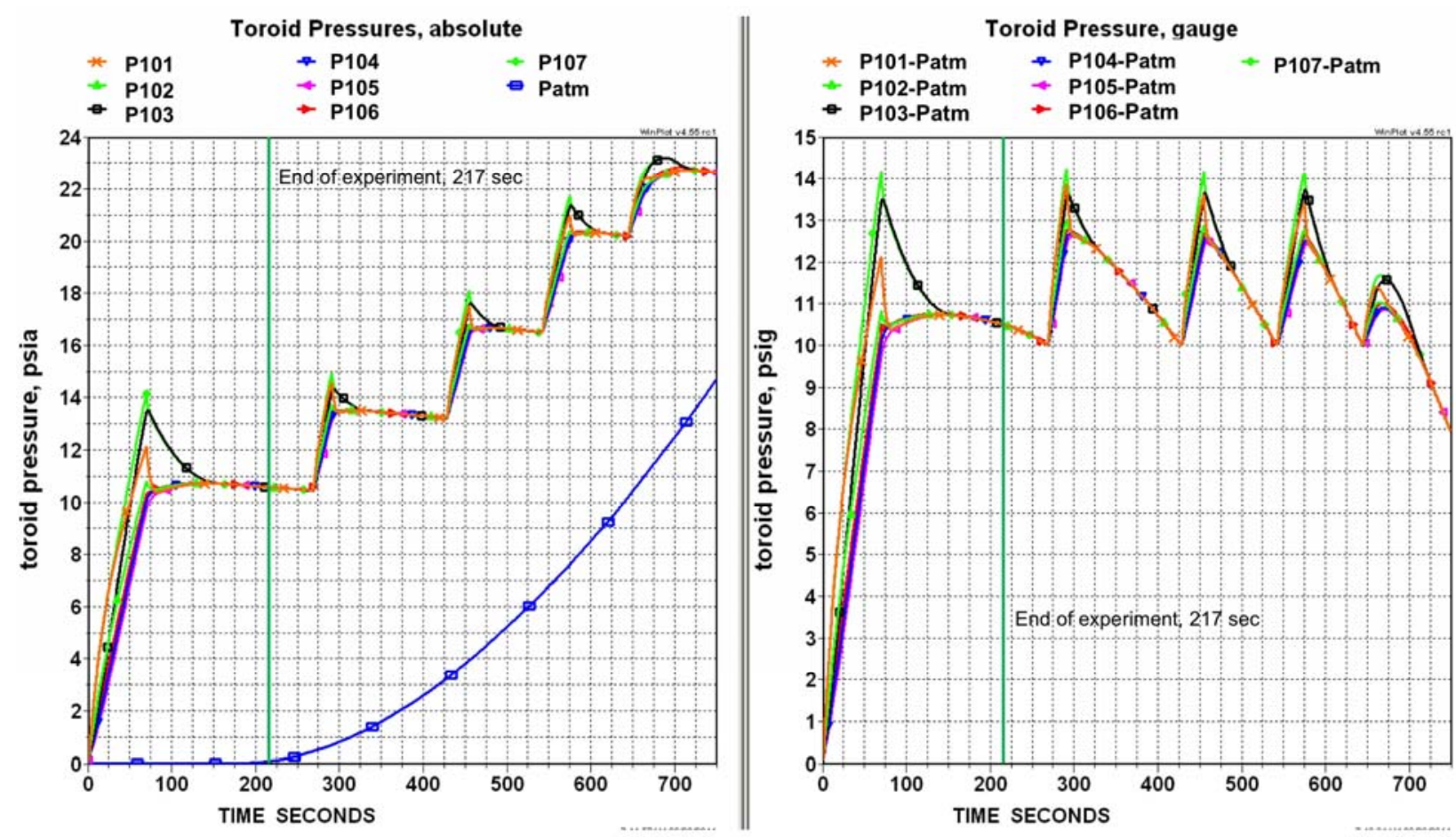

Figure 3.7: Preliminary Toroid and Atmospheric Pressures versus Time

The CG-offset segment is responsible for translating the CG laterally in 1 dimension. The offset CG enables the creation of lift, giving the vehicle the ability to somewhat control it's direction. The CG offset will have the capability to move the CG constantly around during the entire mission experiment window. This portion of the control system will be open loop and follow a predetermined set of commands. IRVE-3's CG-offset segment has the ability to travel +/- 1.5 inches, or alter the CG by +/- 0.75 inches. Minor modifications to IRVE-3's CG will allow the IRVE-4's system to travel +/- 2inches which enables a higher and more flight-like L/D.

The NSROC Inertial Attitude Control System (NIACS) segment performs two main tasks: reorienting the vehicle prior to atmospheric entry and controlling the roll attitude of the vehicle during the PID maneuvers. In order to perform the bank maneuver, the CG-offset system will be left at the extreme offset location and the NIACS will be used to perform the bank maneuver. Most, if not all of the maneuvers performed by the NIACS will be closed loop.

The Telemetry and ballast segments are the last two segments. The Telemetry segment contains everything necessary to telemeter data from the mission down to the ground, sea, or air assets such as the PCM unit, MultiFunction Timers, battery packs S-Band and C-Band Transmitters, antennas for each system and pyro event timer(s). Finally, the ballast segment has been added to IRVE-4 to ballast the CG as far back as possible. Having a further aft CG allow a smaller lateral CG change have a larger impact on the trim angle of attack. This enables the CG-offset mechanism to not have to travel as far to reach the same angle of attack as it would with a less further aft CG. The further aft CG does however have implications on the overall static stability of the vehicle. IRVE-3 is rock solid stable at an X/D of 0.25 . The proposed ballast would only bring IRVE-4's X/D to 0.3, which is still considered a very stable CG location. With the full 2-inch travel of the CG-offset mechanism the lateral CG shifts one inch. This 1-inch of CG travel, enables us to obtain at least 8 degrees angle of attack if this were a rigid body. Due to flexibility of the HIAD and current modeling, we are expecting on the order of 10-11 degrees in angle of attack at max dynamic pressure, resulting in a $\mathrm{L} / \mathrm{D}$ of 0.12 . At the end of the ballast segment are 4 cameras that enable a full look at the back of the HIAD through the whole experiment.

\section{Flight Dynamics}

The goal of the IRVE-4 preliminary trajectory design is to maximize the duration of hypersonic and supersonic environments for system identification activities. The Black Brant $\mathrm{X}$ sounding rocket ascent trajectory can be customized through selection of launch rail elevation angle and stage ignition timing to maximize the effectiveness 
of the IRVE-4 experiment. The maximum duration for performing system identification activities is obtained for the trajectory with the shallowest entry flight path angle that satisfies the ascent and entry vehicle constraints in the presence of uncertainties. The ascent vehicle constraints considered are minimum launch rail elevation, dynamic pressure requirements for stage ignition and jettison, and minimum event durations. The entry vehicle design constrains the optimal trajectory due to the time required for aeroshell inflation and vehicle reorientation prior to entry interface.

Between separation from the launch vehicle and the beginning of inflation, 10 seconds is budgeted for the reentry vehicle to attain sufficient distance from the upper stage and nosecone to prevent recontact. Prior to entry interface, 100 seconds is budgeted to complete inflation, with an additional 40 seconds added to account for reductions in exoatmospheric timeline due to ascent dispersions. Reorientation with the attitude control system will begin once the aeroshell has reached an internal pressure sufficient to maintain a rigid interface between the aeroshell and centerbody and will take place simultaneously with the latter part of inflation. The reorientation event is not expected to increase the total amount of exoatmospheric time required. The nominal experiment window duration resulting from this design process is approximately 60 seconds, defined as the time from $0.5 \mathrm{~g}$ of deceleration to Mach 1.2. The end-to-end trajectory time history and re-entry timeline is shown in Figure 4.1.

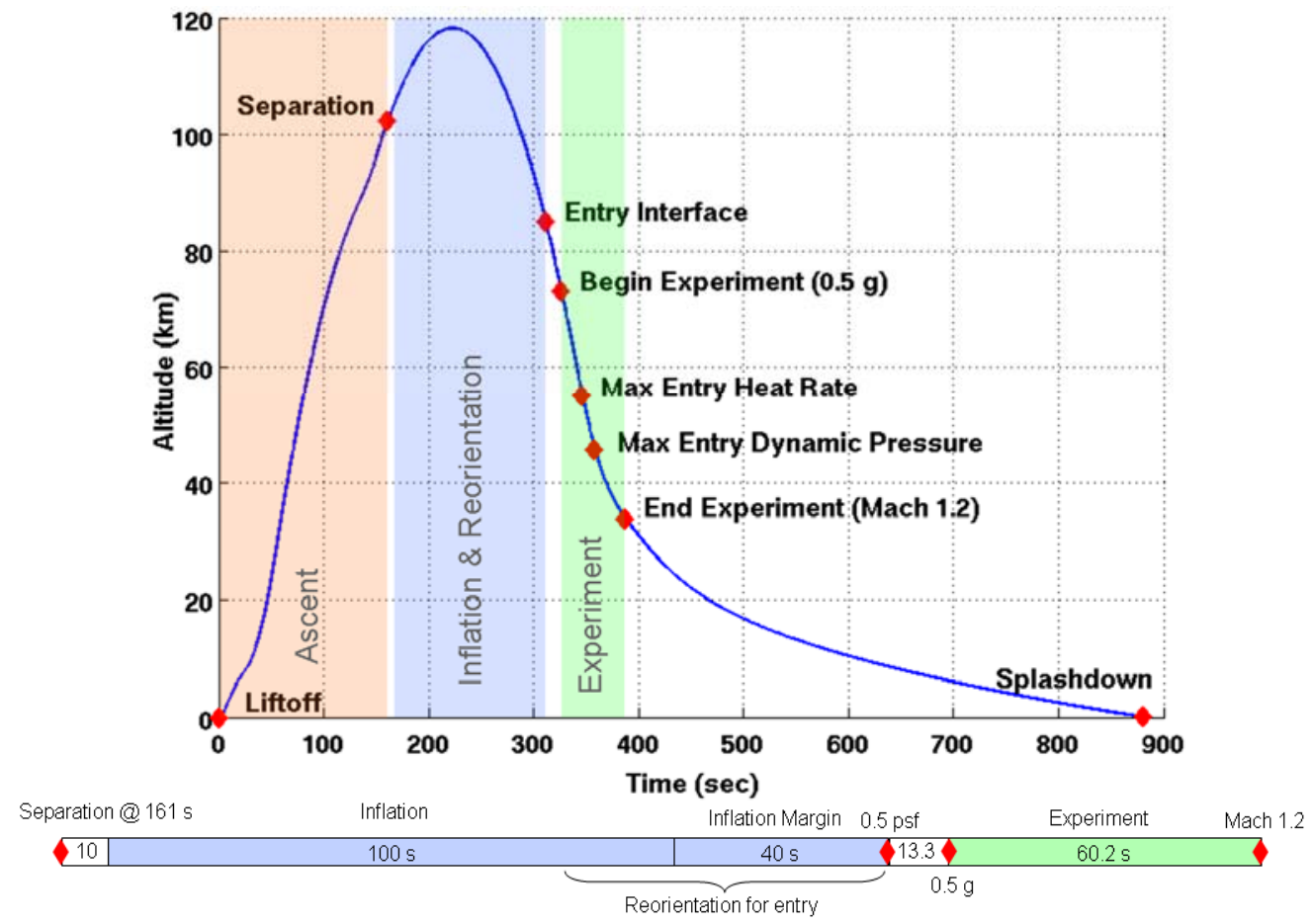

Figure 4.1: Preliminary IRVE-4 Trajectory

The re-entry vehicle trajectory has been modeled with the Program to Optimize Simulated Trajectories II (POST2) $)^{4,5,6}$ to provide inputs for vehicle system design. The POST2 simulation includes attitude control system and lateral CG translation models to evaluate the effects of system identification maneuvers. Six degree-of-freedom, IRVE-3 derived aerodynamic and mass property models are used to propagate the entry vehicle from separation to splashdown. Time histories of Mach number, dynamic pressure, acceleration, and heat flux are co-plotted in Figure 4.2 for the preliminary trajectory. As can be seen the maximum heat rate of 6.8 is lower than IRVE-3's expected 15 $\mathrm{W} / \mathrm{cm}^{2}$. Although the maximum heat rate is lower, the overall heat load is higher, but not high enough to cause any issues. 


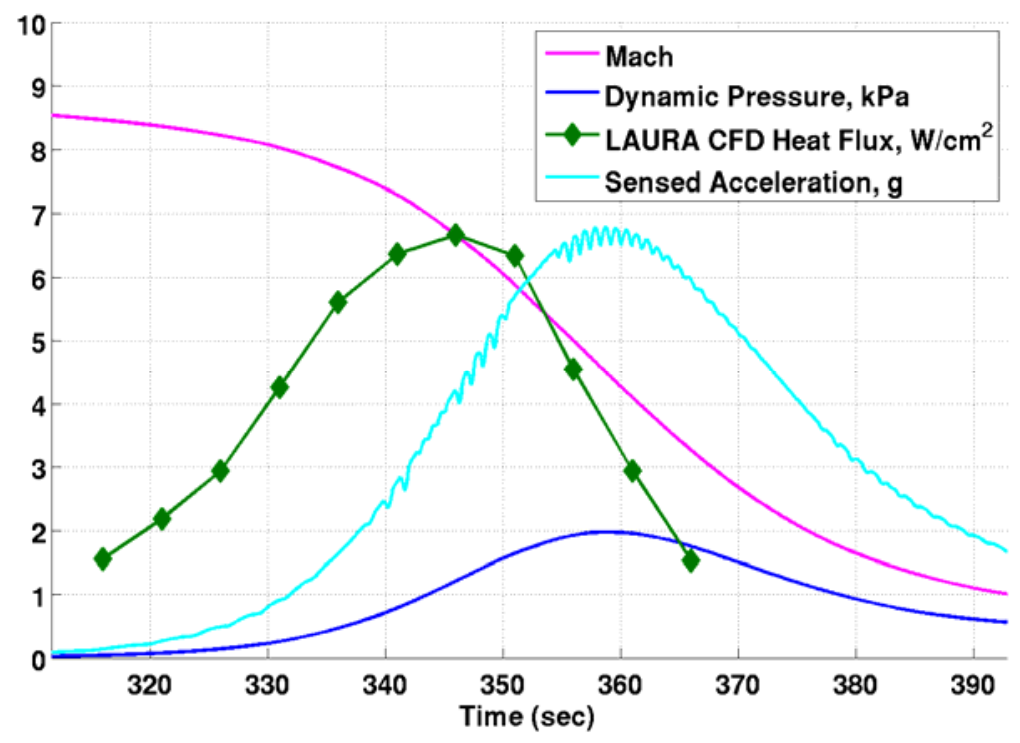

Figure 4.2: Preliminary IRVE-4 Re-entry Conditions

\section{Structural Analysis}

The goal of the structural analysis at this phase of the project was to understand the inflatable structure stiffness so that an assessment could be made of the deformation of the system subjected to aerodynamic reentry pressure. In order to gain confidence in the material property modeling and analysis methods a modal test was conducted on an engineering development article. The test article is shown in Figure 5.1. The test setup consisted of 20 triaxial accelerometers and a single, random input shaker. The center piece of the test article was fixed to the floor.

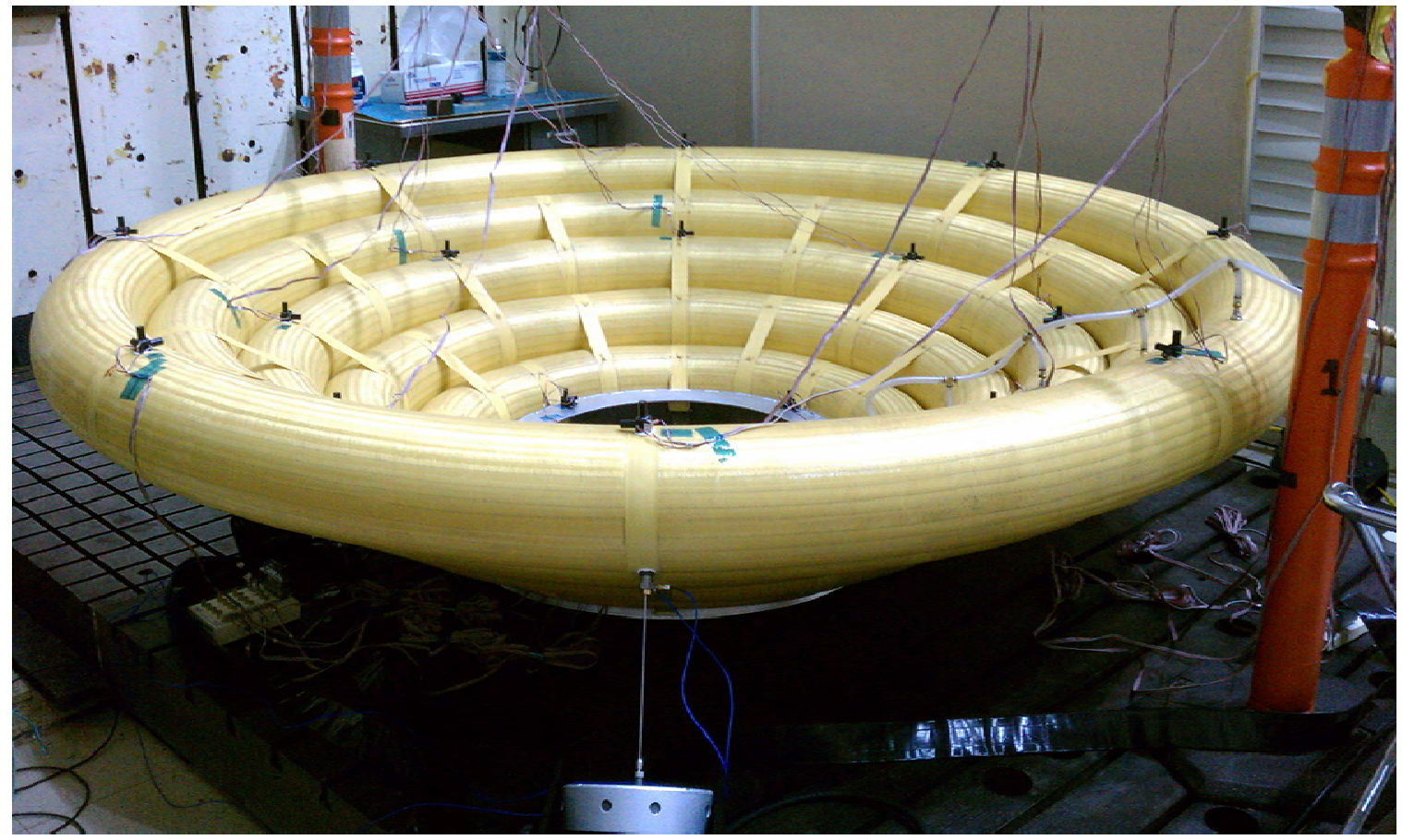

Figure 5.1: Modal Test Setup for the 5-cell Engineering Development Unit 
The test captured the first three system modes. After some minor adjustments to the model the final test/analysis comparison is shown in Figure 5.2 for three different inflation pressures. Mode shapes are shown in Figure 5.3.

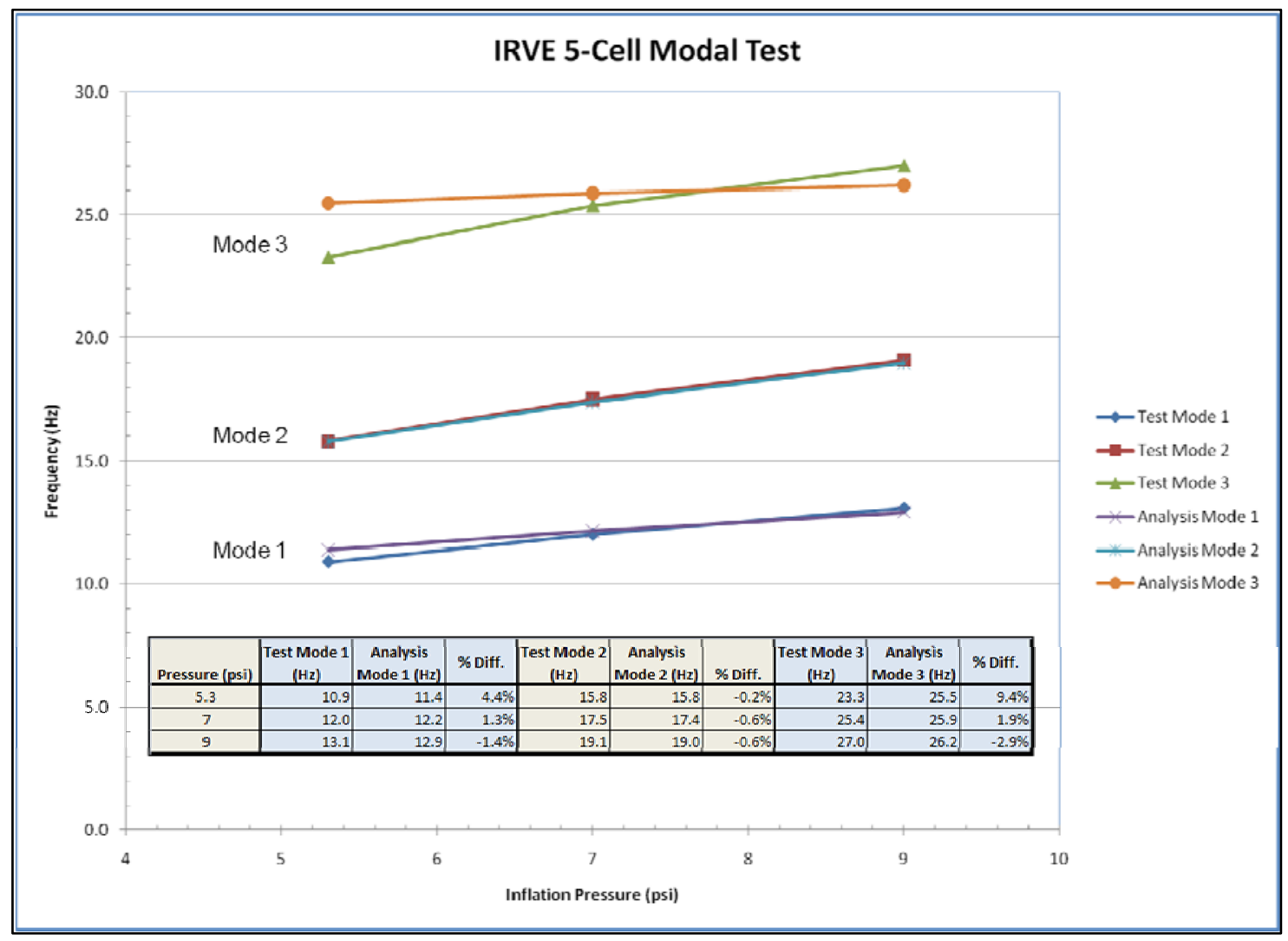

Figure 5.2: Test/Analysis Frequency Comparison

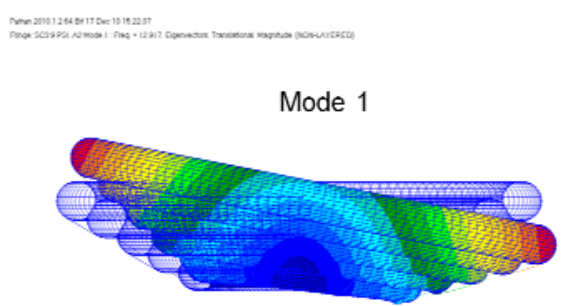

Test: $13.1 \mathrm{~Hz}$

Analysis: $12.9 \mathrm{~Hz}$

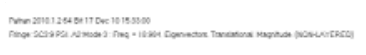

Mode 2

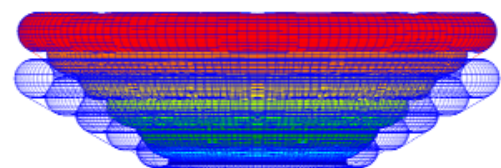

Test: $19.1 \mathrm{~Hz}$

Analysis: $19.0 \mathrm{~Hz}$

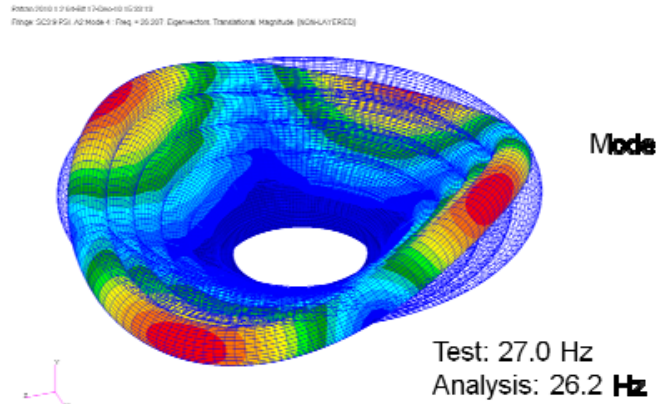

Figure 5.3: Mode Shapes at 9 psi Inflation Pressure

American Institute of Aeronautics and Astronautics 
The correlated material properties were then used in the finite element model of the IRVE-4 flight article. Material properties for the outer thermal protection system (TPS) layer were estimated, assuming minimal contribution to the system stiffness. The IRVE-4 model is shown in Figure 5.4.

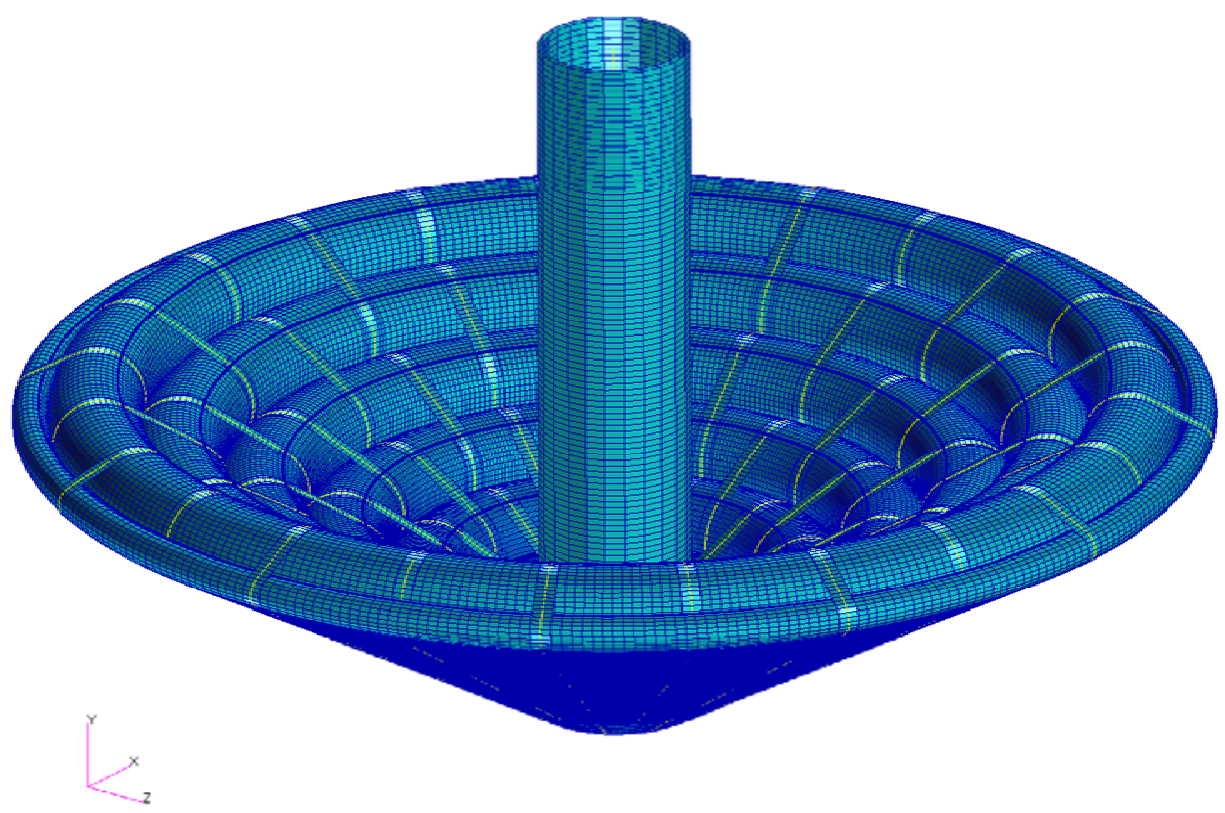

Figure 5.4: IRVE-4 Finite Element Model

The IRVE-4 model was subjected to an aerodynamic pressure profile associated with a 10-degree angle of attack and the resulting deformation was computed. The predicted deformations are shown in Figure 5.5. In the next phase of the project such deformations will then be provided back to flight dynamics to determine any impact on angle of attack. Aerodynamic analysis and structural analysis will iterate until a converged deformed shape is achieved.
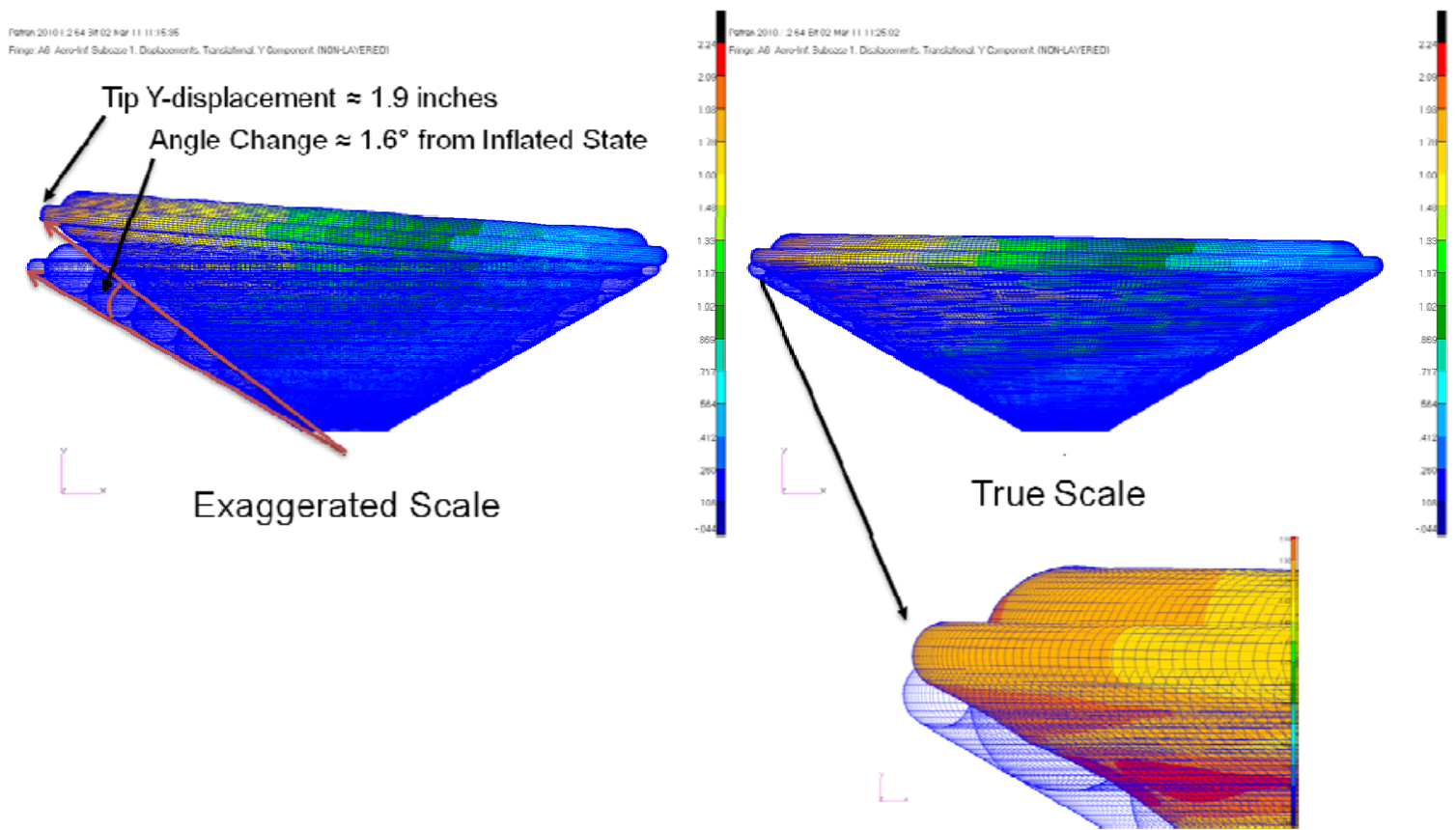

Figure 5.5: IRVE-4 Aeroshell Deformations at 10-degree Angle of Attack

American Institute of Aeronautics and Astronautics 


\section{Planned Maneuvers}

The experimental portion of the IRVE-4 flight has several objectives: evaluating the response of the RV to control inputs, evaluating the aerodynamic performance and stability of the RV, and evaluating the structural response of the RV to control inputs, along with the impact of the structural response on the aerodynamics and the controllability of the RV.

The control inputs that are currently under consideration for this flight include the lateral shifting of the CG to vary the trim angle of attack and $\mathrm{L} / \mathrm{D}$, and bank maneuvering (rolling the RV about the velocity vector) to reorient the lift vector. The CG shifts will be effected by actuation of the CG offset mechanism. The CG offset commands will be pre-programmed and performed as open-loop maneuvers to assess the vehicle response to the CG shift. No closed-loop control of the RV by CG shift will be performed during this mission, although the results could be used to inform the design of such a controller. The RV's cold-gas reaction control system (NIACS) will be used to perform the bank maneuvers and to hold roll attitude at other times during the experiment; this will be accomplished by conventional closed-loop control.

The primary challenges in designing a sequence of test maneuvers for this flight are the short duration of the experimental window (approximately 60 seconds) and the rapidly changing flight conditions (primarily Mach and dynamic pressure, as shown in Figure 4.2). The overall strategy is to perform the two control evaluation maneuvers (CG shift and bank) in the center of the deceleration pulse, when the dynamic pressure is at its highest. Part of the CG shift maneuver will be a test for structural hysteresis at point of zero CG lateral offset. Aerodynamic characterizations will performed both prior and subsequent to this period via a slow CG shift with a frequency sweep superimposed; the first of these will be performed in hypersonic flight conditions (Mach 8 to Mach 7), while the second will be performed supersonically (Mach 3 to Mach 1.7). There will be an additional pulse of the CG shift at low supersonic conditions (about Mach 1.4) to help identify aerodynamic pitch damping. Finally, a set of openloop RCS pulses will be performed at the beginning and the end of the experimental window to excite the first bending mode of the aeroshell.

The general timeline of these maneuvers is shown in Figure 6.1. The initial concept for the CG shift maneuver design is shown in Figure 6.2, and the full design concept of the CG shift for preliminary evaluation is shown in Figure 6.3.

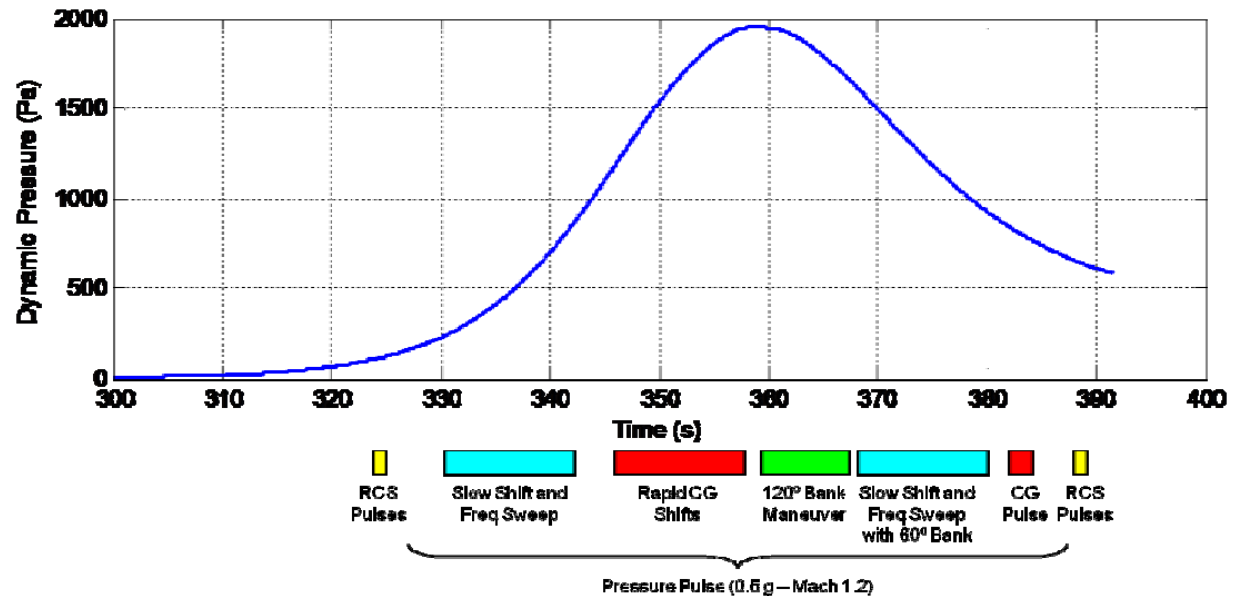

Figure 6.1: Maneuver timeline vs dynamic pressure 
$\alpha$

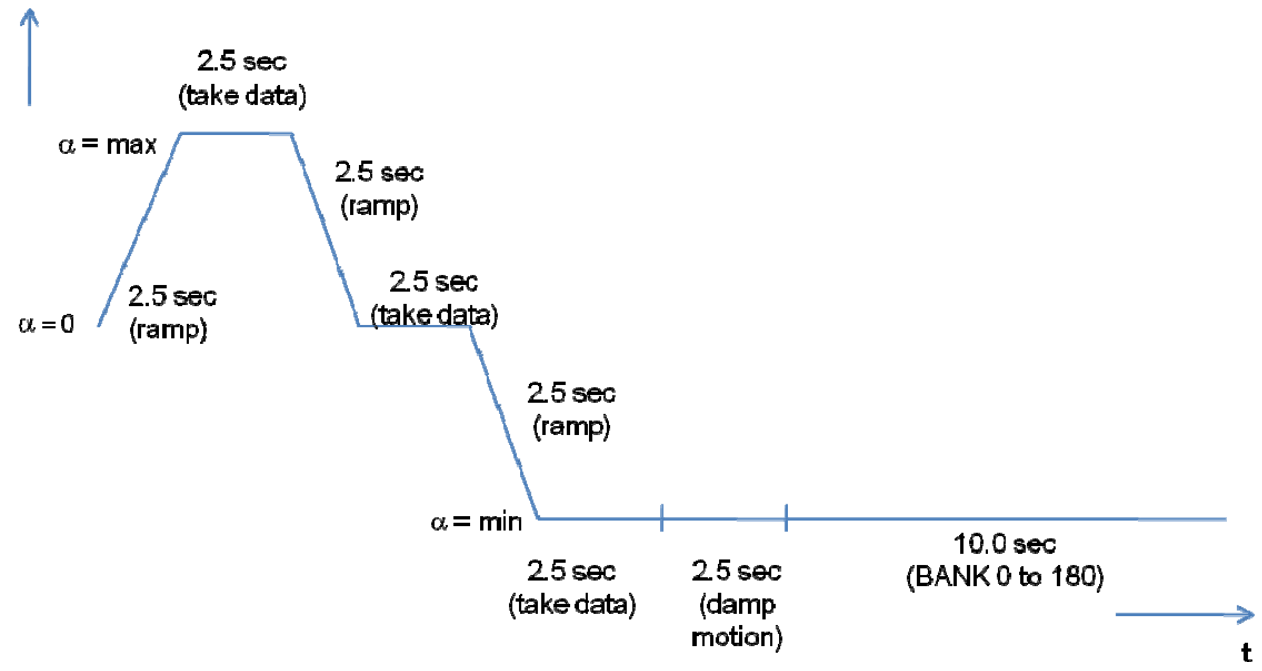

Figure 6.2: Initial concept for CG shift timeline

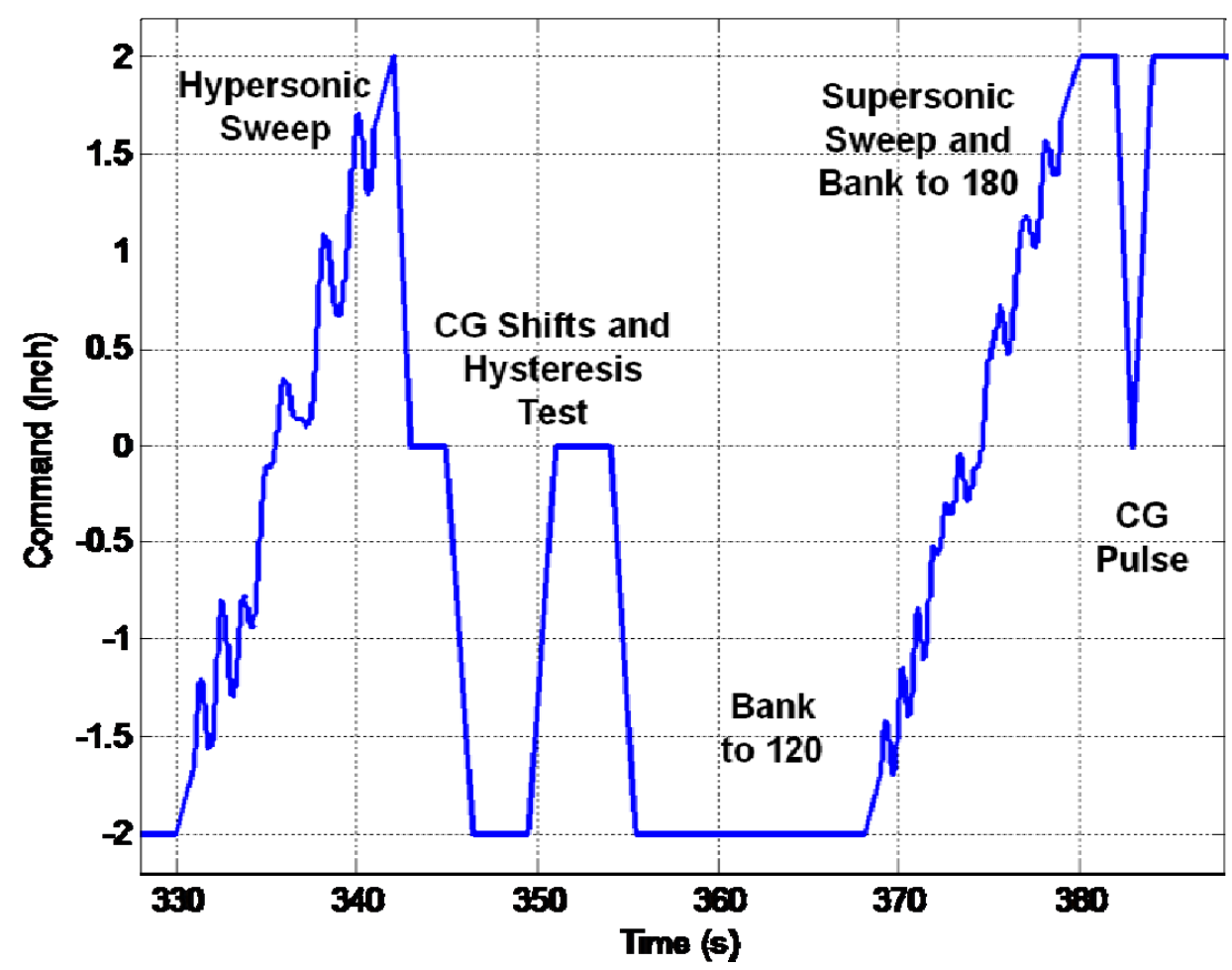

Figure 6.3: Preliminary CG shift design

The execution of the experimental flight maneuvers was evaluated with the Space Transportation Analysis and Research Simulation (STARS), which is a six-degree-of-freedom Simulink ${ }^{\circledR}$-based simulation developed at the NASA Langley Research Center for the Ares I-X program. [Simulink is a registered trademark of The MathWorks, Inc.] The STARS simulation utilizes the same IRVE-3 derived aerodynamic and mass property models as does the POST2 simulation, and it also includes a simple linear model for the first structural bending mode of the aeroshell. Simulations of the experimental portion of the IRVE-4 flight were performed, both with and without the aeroshell bending (flex and rigid). 
Comparison plots for the angle of attack (alpha) and aerodynamic pitch moment coefficient $\left(\mathrm{C}_{\mathrm{m}}\right)$ are shown in Figure 6.4. Notice that the inclusion of the aeroshell bending mode tends to increase the magnitude of the angle of attack, and that the effect is more pronounced with increased dynamic pressure. Post-flight parameter identification (PID) is planned to identify the aerodynamic characteristics of the RV, including the effects of the aeroshell bending, utilizing linear regression methods on equation-error formulations in both time and frequency domains. Preliminary models for the aerodynamic force and pitch moment coefficients have been identified including both linear and non-linear terms; additional terms remain to be identified to account for the bending effects on the aerodynamics.
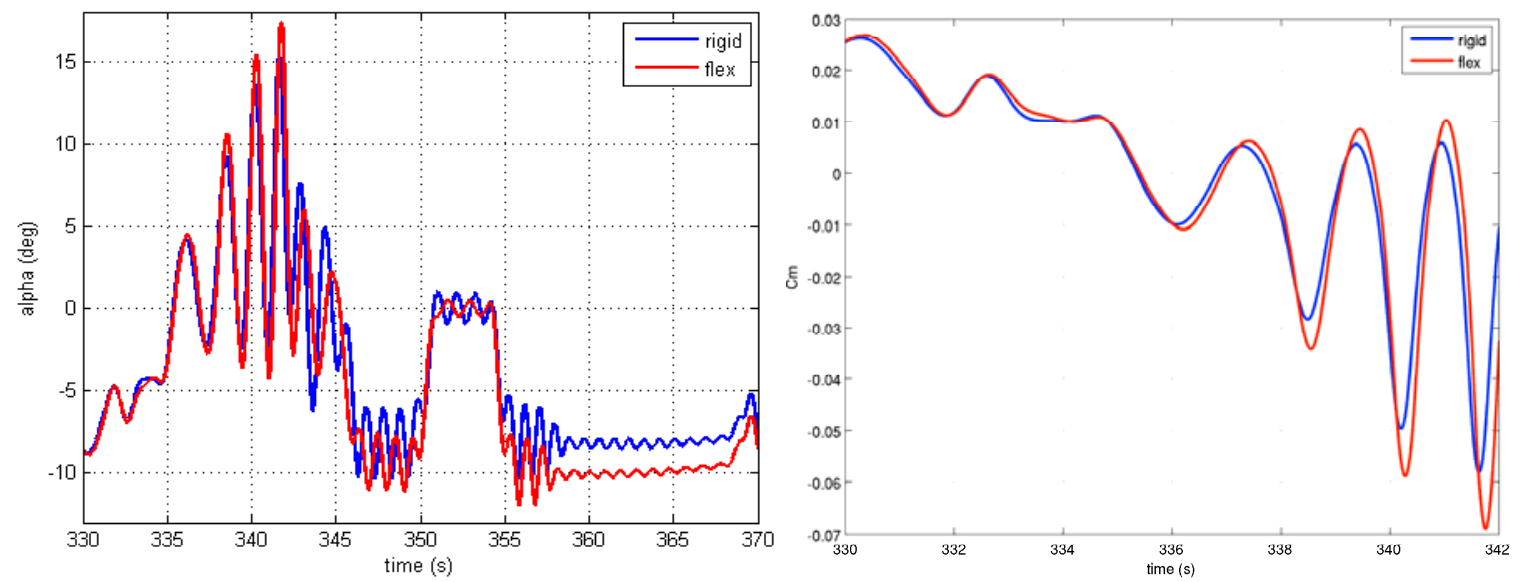

Figure 6.4: Angle of Attack and Pitching Moment Comparison: Rigid vs. flex aeroshell

\section{Conclusion}

IRVE-4 will be an inexpensive, first look at understanding how a HIAD will respond given a set of control inputs. This initial look will be accomplished through a predetermined set of PID maneuvers geared to aide future controlled missions. With this increased understanding of the coupled structural-aerodynamics of HIADs many pitfalls will be avoided in designing an appropriate control systems for the larger, controlled missions. Bank angle control and shifting the CG laterally are the two control strategies that will be focused on during this study. IRVE-3 is currently scheduled to launch in the spring of 2012. Depending on funding, IRVE-4 is proposed to launch in the spring of 2014.

\section{Acknowledgments}

Daniel Litton would like to acknowledge the efforts of the entire IRVE-4 team at NASA Langley, NASA Wallops, and NASA JSC. In addition to the authors listed direct contributions to this paper or the project come from John Rogers (LaRC), Todd Ferrante (LaRC), Daniel Matz (JSC), Ron Sostaric (JSC), Chris Cerimele (JSC), Eric Queen (LaRC), Bethany Schiller (LaRC), Walt Bruce (LaRC), Warren Davis (LaRC), Dave Christhilf (LaRC), Jeff Massie (LaRC), and David Kilcoyne (NSROC).

\section{References}

${ }^{1}$ Braun, R., and Manning, R., “Mars Exploration Entry, Descent and Landing Challenges,” IEEE Paper 2006-0050781.

${ }^{2}$ Axdahl, E., Cruz, J. R., Schoenenberger, M. and Wilhite, A., "Flight Dynamics of an Aeroshell Using an Attached Inflatable Aerodynamic Decelerator,” AIAA Paper 2009-2963, 2009.

${ }^{3}$ O’Keefe, S. A., Bose, D. M., "IRVE-II Post-Flight Trajectory Reconstruction,” AIAA Atmospheric Flight Mechanics Conference, AIAA Paper 2010-7515, 2009.

${ }^{4}$ Bauer, G.L., Cornick, D.E., and Stevenson, R., "Capabilities and Applications of the Program to Optimize Simulated Trajectories (POST),” NASA CR-2770, February 1977.

${ }^{5}$ Striepe, S.A., et al, “Program to Optimize Simulated Trajectories (POSTII), Vol. II Utilization Manual,” Version 1.1.6.G, January 2004, NASA Langley Research Center, Hampton, VA.

${ }^{6}$ Striepe, S.A., Aguirre, J.T., Fisher, J.L., et al, "Program to Optimize Simulated Trajectories (POSTII), Vol. I Guide for New Users,” Version 1.1.6.G, November 2003, NASA Langley Research Center, Hampton, VA. 